\title{
Subtle order in settling suspensions*
}

\author{
B. J. Ackerson ${ }^{\ddagger}$, X. L. Lei, and P. Tong \\ Department of Physics, Oklahoma State University, Stillwater, OK 74078-3072, USA
}

\begin{abstract}
A dilute suspension of uniform, non-Brownian spheres settles slowly in a viscous solvent. The initially well-mixed system showing Poisson or random occupancy statistics evolves to a system having reduced number fluctuations, but otherwise appearing random. The reduced number fluctuations are consistent with recent measurements of velocity fluctuations in settling suspensions. These experimental results test the assumptions leading to the theoretical predictions by Calflisch and Luke that the velocity fluctuations increase without limit with increasing sample dimension. The theoretical prediction assumes Poisson occupation statistics contrary to our observations.
\end{abstract}

\section{INTRODUCTION}

Fluctuations play an important role in our understanding of the statistical mechanics of many-bodied systems including solution chemistry. The notion of statistical independence is an elementary idea that undergirds these understandings [1]. For example, consider a homogeneous many-body system, either an equilibrium or nonequilibrium system. Partition the system into $M$ cells of equal dimension. The dimension should be greater than or equal to any spatial correlation length within the system. The average value of an extensive quantity $f$ in a total volume containing $M$ cells is determined by sampling each region (to determine $f_{i}$ for the $\mathrm{i}^{\text {th }}$ region) and adding up the measured values (summing over i). Alternatively, the average for a volume containing $M$ cells will be $M\left\langle f_{i}\right\rangle$, where $\left\langle f_{i}\right\rangle$ is the average value for the $\mathrm{i}^{\text {th }}$ cell. The mean-square fluctuation about the average is

$$
\begin{aligned}
\left\langle\Delta f^{2}\right\rangle & =\left\langle(f-\langle f\rangle)^{2}\right\rangle=\left\langle\sum_{i}\left(f_{i}-\left\langle f_{i}\right\rangle\right) \sum_{j}\left(f_{j}-\left\langle f_{j}\right\rangle\right)\right\rangle \\
& =\left\langle\sum_{i}\left(f_{i}-\left\langle f_{i}\right\rangle\right)^{2}\right\rangle=M\left\langle\Delta f_{i}^{2}\right\rangle
\end{aligned}
$$

where $\left\langle\Delta f_{i}^{2}\right\rangle$ is the mean-square fluctuation in the $\mathrm{i}^{\text {th }}$ region. Because the different cells are larger than the spatial correlation range for the system, the third equality above is zero when averaging different cells. Different cells are statistically independent such that

$$
\left\langle\left(f_{i}-\left\langle f_{i}\right\rangle\right)\left(f_{j}-\left\langle f_{j}\right\rangle\right)\right\rangle=\left\langle\left(f_{i}-\left\langle f_{i}\right\rangle\right)\right\rangle\left\langle\left(f_{j}-\left\langle f_{j}\right\rangle\right)\right\rangle=0
$$

Thus, the fluctuation for the sample containing $M$ identical and independent cells is $M$ times the fluctuation for a single cell.

A number of consequences follow from the assumption of "statistical independence" of the cells. Firstly, Prof. Lynden-Bell [2] calculates via molecular simulation the equilibrium energy associated

*Plenary lecture presented at the $27^{\text {th }}$ International Conference on Solution Chemistry, Vaals, The Netherlands, 26-31 August 2001. Other presentations are published in this issue, pp. 1679-1748.

¥Corresponding author 
with a solute molecule in a solvent. Accurate results require using system sizes that are on the order of correlation length to calculate a system energy $E$ with and then without the solute molecule. The energy attributed to the solvent molecule is the difference of the two calculated energies. When large numbers of solvent molecules are used, say containing $M$ correlation cells, then the uncertainty in the calculated energy increases as $\sqrt{M}$ because

$$
\sqrt{\left\langle\Delta E^{2}\right\rangle}=\sqrt{M} \sqrt{\left\langle\Delta E_{i}^{2}\right\rangle}
$$

The error grows as the root of the number of correlation regions included. The energy fluctuations for large systems "wash out" any calculated energy differences between systems with and without the solute molecule.

Prof. Angell described nonequilibrium, glassy systems [3]. While he did not discuss colloidal glasses explicitly, these glasses also show nonergodic behavior with slowly decaying fluctuations. Here, precise measurements require extensive spatial averaging over a large number of correlation regions [4]. Average measurements improve with increased number of correlation cells, because the relative fluctuation to mean decreases.

$$
\frac{\sqrt{\left\langle\Delta f^{2}\right\rangle}}{\langle f\rangle}=\frac{\sqrt{M\left\langle\Delta f_{i}^{2}\right\rangle}}{M\left\langle f_{i}\right\rangle}=\frac{1}{\sqrt{M}} \frac{\sqrt{\left\langle\Delta f_{i}^{2}\right\rangle}}{\left\langle f_{i}\right\rangle}
$$

This well-known result forms the foundation for signal averaging. For an increasing number of independent measurements added together, the signal grows faster than the noise (fluctuations). This argument for increased precision is quite different from that associated with eq. 3, yet both rely on statistical independence and show an $\sqrt{M}$ dependence.

Finally, in the poster presentation by Kagan et al., they describe concentration fluctuations in equilibrium mixtures of alkali-metal liquids. The static structure factor $S(k)$ measured by scattering techniques estimates microscopic pair correlations within the system. In the small wave-vector, long wavelength limit $S(0)$ is a thermodynamic quantity independent of sample size and related number and concentration fluctuations. For simple single component fluids we have

$$
\frac{\left\langle\Delta N^{2}\right\rangle}{\langle N\rangle}=S(0)
$$

Here $N$ measures the number of particles in the sample volume. Reflection on the earlier arguments reveals that this quantity should be independent of the number of correlation regions $M$. Therefore, it is a thermodynamic quantity, provided the measurement volume is greater than the spatial correlation range. Inter-particle repulsive forces tend to reduce $S(0)$ and, therefore, the mean-square fluctuations as compared to attractions, which do the opposite. An example of enhanced fluctuations occurs near the thermodynamic critical point where attractions lead to large-scale particle number fluctuations. Alternatively, the hard-sphere fluid shows reduced fluctuations with increasing hard-sphere concentration. Yet, despite reduced or enhanced fluctuations, the mean-square fluctuation is proportional to the average number in the sample volume, the larger the sample the larger the mean square fluctuations.

For noninteracting particles, an ideal gas or a dilute hard-spheres system, the long wavelength limit of the static structure factor is unity $[S(0)=1]$, and the mean-square number fluctuation then equals the average particle number. The Poisson distribution satisfies the same relationship and describes the statistics of points randomly positioned in space. The Poisson distribution presented below

$$
P(N)=\frac{e^{-N /\langle N\rangle}}{N !}
$$


gives the probability for finding $N$ particles in a region having an average number $\langle N\rangle$.

The statistical independence assumption is deeply embedded in our thinking concerning equilibrium and nonequilibrium many-body systems and systems of "randomly" positioned particles. When a many-body "random" system fails to exhibit statistical independence it is unusual and surprising. Consider the two distributions of points in Fig. 1. One of the distributions is random, and one is not. Can you decide which is random?

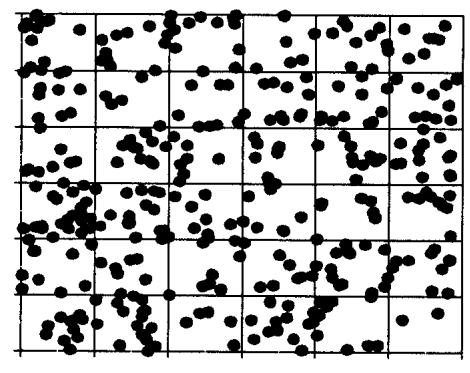

a

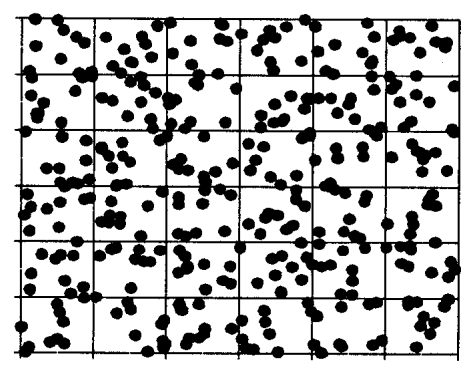

b

Fig. 1 A random number generator produces both figures above. For (a), all 360 points are placed at random in the whole region. In (b), 10 points are placed randomly in each of the 36 squares. Thus, each small square in both figures has an average of 10 points. Figure 1a has the expected random fluctuation about the average $\left\langle\Delta N^{2}\right\rangle \sim\langle N\rangle \sim 10$, but Fig. 1b has no fluctuations about the average $\left\langle\Delta N^{2}\right\rangle=0$.

The untrained eye typically selects Fig. $1 \mathrm{~b}$ as random because it appears a little more uniform and lacks the clustering observed in Fig. 1a. Observers assume the clustering is due to some nonrandom process. Yet, both distributions look fairly random. However, the clustering in Fig. 1a is a result of random fluctuations, those that are described by the Poisson distribution. The distribution on the right is highly nonrandom because each box contains exactly 10 points. There are no large-scale number fluctuations in this distribution. It is indeed more uniform, much more uniform. Locally, both distributions are random, so measures of order, like the pair correlation function, may prove useless to characterize this subtle order. Yet, a change in order from that given in Fig. 1a to that in Fig. 1b is what we observe for the distribution of particle positions in settling suspensions.

The settling of a suspension of monodisperse particles received renewed scrutiny with the publication of a short article by Calflish and Luke [5]. While Batchelor [6] had earlier calculated the average particle settling velocity, Calflish and Luke calculated the fluctuations about the average velocity utilizing the same assumptions [7]: (i) a low-particle Reynolds number or the neglect of inertia, (ii) twobody hydrodynamic interactions, (iii) a random particle distribution in space, and (iv) the system size infinite in the direction transverse to the settling direction. Batchelor's result found experimental support, but the calculation of Calflish and Luke predicted a divergence of the velocity fluctuations as the sample container linear dimension becomes large.

Segre' et al. [8] provide intuition through an argument similar in spirit to Hinch [9]. This argument suggests the Calflish and Luke divergence is rooted in the statistical independence assumption. Consider a region of suspension of linear dimension $l$. The average number of particles is given by $\langle N\rangle=l^{3} \phi / v_{p}$, where $\phi$ is the particle volume fraction, $a$ is the particle radius, and $v_{p}=4 \pi a^{3} / 3$ is the particle volume. Random Poisson statistics imply a fluctuation from the average number of order $\sqrt{\left\langle\Delta N^{2}\right\rangle}=\sqrt{\langle N\rangle}$ and corresponding weight deviation of order $\sqrt{\langle N\rangle} \Delta \rho g v_{p}$, where $\Delta \rho$ is the difference in particle and solvent densities and $g$ is the acceleration of gravity. When Stokes drag of order $6 \pi \eta l \Delta U$ balances the excess weight, the typical velocity fluctuation $\Delta U$ becomes $\Delta U / U_{o}=\sqrt{N} a / l=$ $\sqrt{3 \phi l / 4 \pi a}$. Here, $\eta$ is the shear viscosity and $U_{o}=2 \Delta \rho g a^{2} / 9 \eta$ is the dilute limit-settling rate. The result corresponds with the Calflisch and Luke result having the same $l$ dependence. The divergence 
here is rooted in the assumed particle statistics. No divergence obtains, for example, if $\left\langle\Delta N^{2}\right\rangle /\langle N\rangle=$ $\langle N\rangle^{-1 / \alpha}$ when $\alpha \geq 3$. However, the particle number occupancy statistics are radically modified from random statistics.

The velocity fluctuation divergence predicted by Calflisch and Luke received much attention. Computer simulations by Ladd [10-11] relax assumptions (ii) and (iii) since they approximate many particle hydrodynamics, and the system configuration may evolve via hydrodynamic interactions away from initially randomized configuration. Yet, the results indicate increased velocity fluctuations with increased sample size. The simulations assume periodic boundary conditions. There is no settling interface nor cell bottom boundary. Experiments, on the other hand, do evidence limited velocity fluctuations. Both indirect measurements of the diffusion of a tracer particle [12-14] and direct measurements of velocity fluctuations [8] have been made.

Koch and Shaqfeh [15] gave the first explanation for the absence of divergent velocity fluctuations in terms of the subtle order revealed in Fig. 1. They postulate a violation of assumptions (ii) and (iii) where three-body hydrodynamic interactions lead to a net deficit of one particle around any reference particle within a correlation range $\xi$. This condition drives the number fluctuations to zero beyond the correlation length, but the predicted correlation length scaling as $\xi=a / \phi$ does not agree with experiments. Other theories are based on a coarse-grained two-fluid description of the suspension. These theories may [17] or may not [16] address assumption (iii) directly through calculation of a static structure factor, but they do produce the experimentally observed correlation length dependencies. Another twofluid theory attributes the Caflisch and Luke divergence to neglect of the finite propagation time of hydrodynamic disturbances. Keeping time-dependent terms then screens the long-range effects [18]. This effect is too weak to predict the suppression seen experimentally. Another possibility is the development of a stable density stratification [19] that quenches velocity fluctuations. Of course, this becomes a time-dependent effect that requires longer and longer periods of time to develop as the system size increases. Finally, Brenner [7] considers the effect of walls, thus relaxing assumption (iv) while maintaining assumption (iii), to produce the experimentally observed correlation length dependence. $\mathrm{He}$ argues that wall effects dominate the experiments in larger sample cells. Eventually, however, increased velocity fluctuations increase particle mixing and suppress number fluctuations, quenching the velocity fluctuations themselves.

Typical measures of suspension structure utilize scattering techniques to measure the static structure factor $S(k)$ or direct imaging to measure the pair correlation function $g(r)$. While long-ranged order and suppression of fluctuations may be observable using these methods, it may not be the best approach. The calculations of Koch and Shaqfeh [15] indicate a long-ranged behavior of $g(r)$ inversely proportional to distance. The deviation of $g(r)$ from unity (the expected value for a dilute random system) may be too small for detection, yet a volume integral for this pair correlation function diverges. Since number fluctuations equal an integral of the pair correlation, they prove to be a sensitive measure of subtle changes in order. Smith [20] measured number fluctuations several decades ago, but the measurement test volumes proved too small to observe significant effects. Recently, we measured number fluctuations [21], and this communication expands on the results presented in that short letter.

\section{EXPERIMENTS}

A vertical rectangular cell having dimension $1 \times 1 \times 4 \mathrm{~cm}^{3}$ contains the samples. We place this cell in a large stirred water bath controlled at $23 \pm 0.1^{\circ} \mathrm{C}$ to eliminate convection produced by temperature gradients. Without such stirring, the suspension-supernatant interface becomes unsettled. With stirring, the interface remains sharp and moves downward at constant rate. A Coherent VERDI laser $(\lambda=532 \mathrm{~nm})$ is used to illuminate the sample. The laser beam passes through a rapidly rotating glass disk, which we lightly dapple with a translucent salve, to produce incoherent light. Then, a horizontal glass rod expands the beam vertically. Finally, a long focal length cylindrical lens forms a uniform, bright, vertical, light 
sheet of thickness $0.35 \mathrm{~mm}$ at the cell center. A normal to the sheet is also normal to one of the faces of the sample container and parallel to the line of sight of the imaging system. Typically, a $4.5 \times 3.4$ $\mathrm{mm}$ patch of this cross-section is imaged through a Leica MZ8 tele-microscope onto a CCD, where images are captured and stored for further processing.

Samples contain polystyrene spheres having diameter $d=41.88 \pm 0.82 \mu \mathrm{m}$ (Bangs Labs, Inc.) and suspended at volume fraction $\phi=0.004$ in polyalkylene glycol (PAG). PAG is an organic Newtonian fluid (automobile air-conditioner lubricant) with density $\rho=0.95 \mathrm{~g} \mathrm{~cm}^{-3}$ and viscosity $\eta=0.092 \mathrm{PaS}$ at $23{ }^{\circ} \mathrm{C}$. Thus, the sample cell viscous damping time is order $1 \mathrm{~s}$. The Stokes velocity is $U_{o}=2 a^{2} \Delta \rho \eta / 9 \sim 0.85 \mu \mathrm{m} \mathrm{s}^{-1}$, giving a total settling time of order $10 \mathrm{~h}$ and a horizontal mixing time based on estimates of $\Delta U$ from 2.5 to $5 \mathrm{~h}$. The Peclet number given by $P e=U_{o} a / D_{o}>10^{6}$, where $D_{o}$ is the Stokes-Einstein diffusion constant [22], means settling dominates Brownian motion. The Reynolds numbers based on particle radius is $\operatorname{Re}=2 a U_{o} \rho / \eta \sim 10^{-7}$ and on maximum cell dimension is $R e=2 L U_{o} \rho / \eta \sim 10^{-4}$, implying negligible inertial effects or the creeping flow limit.

We determine particle configurations by imaging the specular reflection of incident light from each particle in the light sheet. The intensity maxima are not centered on particles, but are offset the same amount for each particle. Figure 2 shows particle configurations shortly after mixing and also late in the settling process. Immediately after mixing and extending for several hours, this slow settling exhibits a turbulent-looking velocity field as seen in Fig. 2b. Combining five images taken at 30 -s intervals produces this "streak" or time-lapsed image. Figure 2a shows a single image from this early stage of settling. Figure $2 \mathrm{~d}$ shows another time-lapsed image taken during the later stages of settling, and Fig. $2 \mathrm{c}$ shows a single image from this sequence. While the single images $2 \mathrm{a}$ and $2 \mathrm{c}$ show similar random-looking configurations of particles, the corresponding velocity fluctuations in $2 \mathrm{~d}$ are less violent than those in $2 \mathrm{~b}$. Evidently, there are subtle configuration differences between the two times.
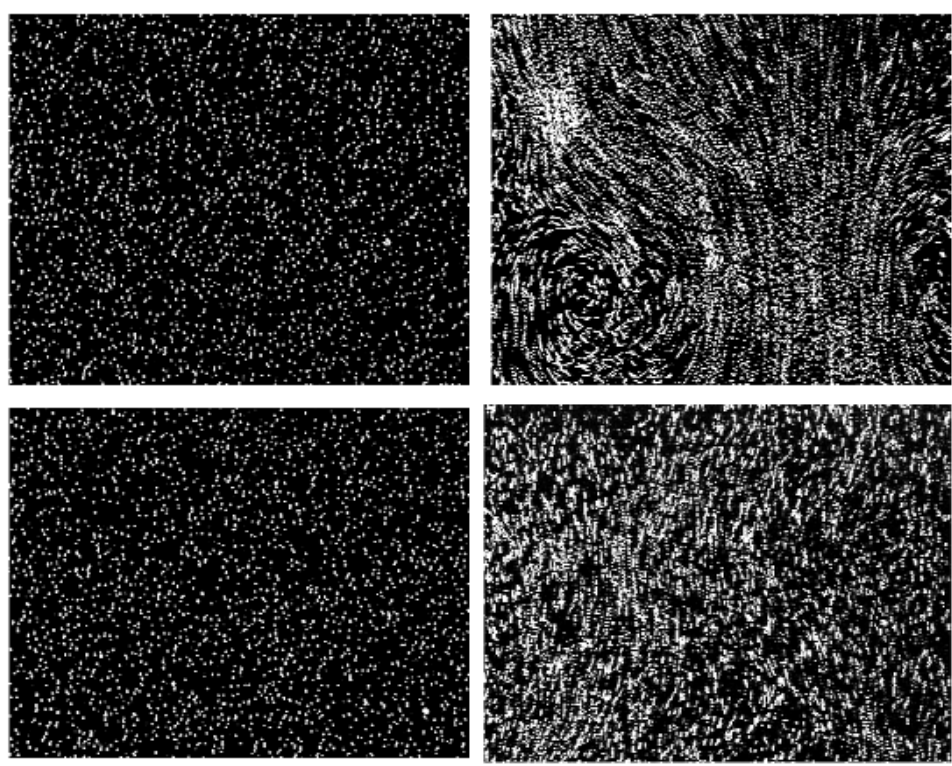

Fig. 2 Images taken during particle sedimentation: (upper left) a particle configuration taken 35 min after mixing, (upper right) a superposition of five sequential images taken at 30-s intervals beginning 30 min after mixing, (lower left) a particle configuration taken $5 \mathrm{~h}$ after mixing, and (lower right) a superposition of five sequential images taken at 30-s intervals beginning $5 \mathrm{~h}$ after mixing. The display area is approximately $4.5 \times 3.4 \mathrm{~mm}$. 
In order to study these particle configurations quantitatively, we determine the particle occupancy distribution by counting the number of particles within a circle of fixed area. A distribution results from randomly positioning the circle 100 to 200 times in each of 50 independent images, obtained by viewing different regions of the light sheet in the lower third of the sample and by changing the depth of the light sheet within the sample. The time duration spanned by the 50 images is $2 \mathrm{~min}$. Measurements of the particle concentration remained constant within experimental error (number fluctuations) in both space and time. For experimental illumination reasons, we limited our viewing volume to the central third of the cell, away from the walls. Therefore, the particles in the observed region should be interacting with "strong" hydrodynamic interactions according to Brenner's reasoning [7] and not be influenced by wall effects.

\section{RESULTS}

Figure 3 shows the occupancy distributions generated for two different sampling areas, corresponding to $\langle N\rangle=6$ and 58. The solid lines show the Poisson distribution for these average occupancy values. This distribution describes the earliest measurements after mixing, but not at the later times, when the experimental distribution narrows relative to the Poisson distribution. Poisson statistics properly apply to point particles and ignore any particle interaction effects, such as the excluded volume for hard spheres. For hard spheres with $\phi=0.004$, solution of the Percus-Yevich equation [23] gives $\left\langle\Delta N_{\text {Hard Sphere }}{ }^{2}\right\rangle=0.97\left\langle\Delta N_{\text {Poisson }}{ }^{2}\right\rangle$, indicating negligible excluded volume effects when comparing the early time distribution with the Poisson distribution.
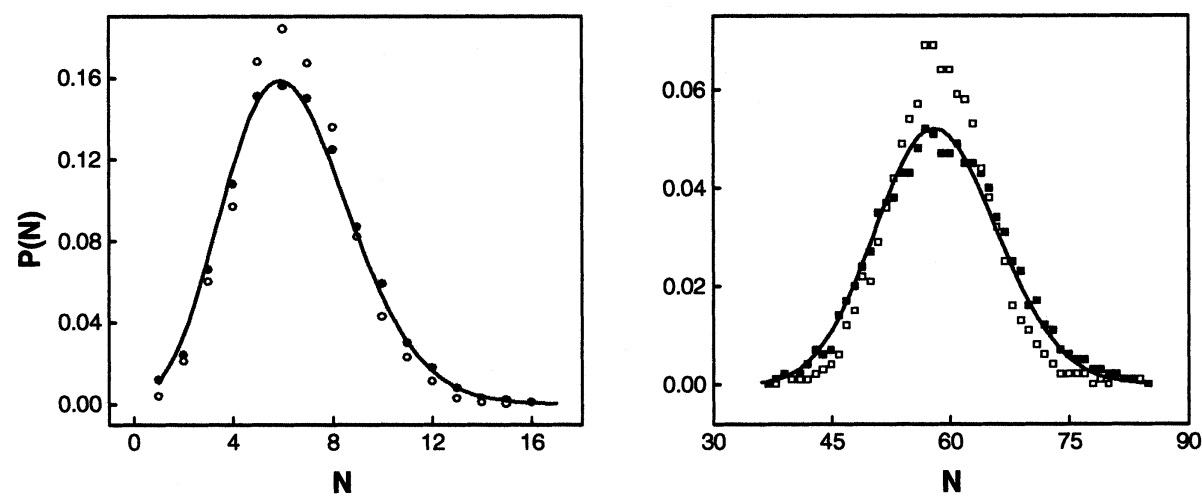

Fig. 3 Particle occupancy distributions: (a) for $\langle N\rangle=62$ min after mixing (filled circles) and $8 \mathrm{~h}$ after mixing (open circles), and (b) for $\langle N\rangle=582 \mathrm{~min}$ after mixing (filled squares) and 8 hours after mixing (open squares).

Figure 4 shows the mean square number fluctuations divided by the mean, a "thermodynamic function" as described for eq. 5, plotted as a function of the average number of particles in the test volume. The initial measurements near unity indicate Poisson statistics, but the number fluctuations become smaller with elapsed time and increased test volumes. The hard sphere volume fraction must be increased by more than an order of magnitude to produce the suppression of number fluctuations observed in the late stages of settling. Such comparisons implicitly assume $\left\langle N^{2}\right\rangle /\langle N\rangle$ is constant and contrary to the $\langle N\rangle$ dependent data shown in Fig. 4. Furthermore, the relaxation time for the decay of the initial random fluctuations is a large fraction of the total settling time and is of the order of the previously estimated horizontal mixing time. This time corresponds with our observations and serves as a caution when assuming density or velocity fluctuation measurements are in steady state. 


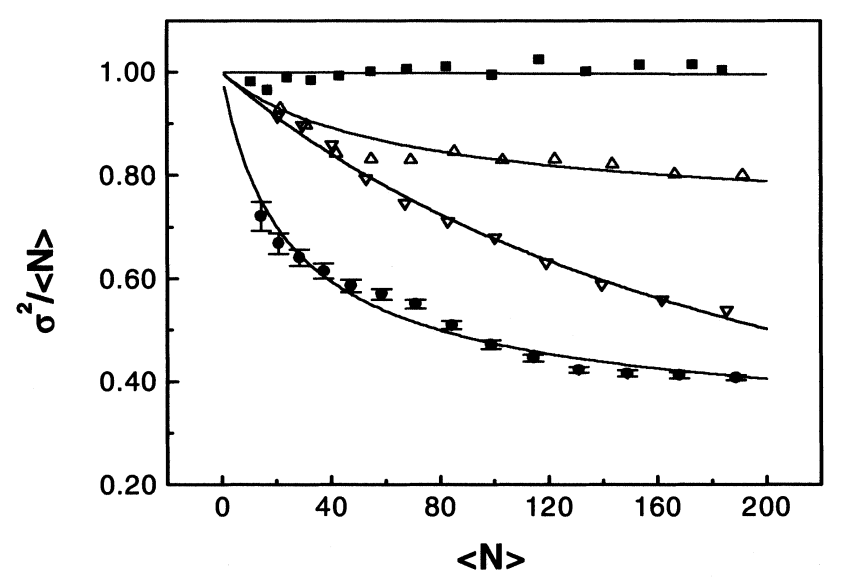

Fig. 4 Reduced number fluctuations, $\left\langle\Delta N^{2}\right\rangle /\langle N\rangle=\sigma^{2} /\langle N\rangle$, as a function of the average number of particles in the test volume, $\langle N\rangle$, shown for different times after mixing: 2 min (solid squares), $4 \mathrm{~h}$ (up triangles), $6 \mathrm{~h}$ (down triangles), and $8 \mathrm{~h}$ (solid circles). The solid curves from top to bottom represent Gaussian theory for $\omega=2$ and $\xi / a=200,33,68$, and 20 and $S(0)=0,0,-7$, and -0.53 , respectively. The error bars represent $68 \%$ confidence estimate of the average value.

\section{DISCUSSION}

While the principle of the experimental methods is straightforward, accurate measurements require care. Some experimental artifacts reduce number fluctuations compared to the Poisson prediction. These artifacts include finite test volume to sample volume effects. Poisson statistics hold in the limit of vanishing test volume to sample volume ratios. Obviously, when the test volume is the sample volume, the total number of particles is fixed and there are no number fluctuations. The test volume must sample a small number of the total particles to avoid suppression of number fluctuations. When particles in the foreground obscure or hide particles in the background, number fluctuations again are reduced artificially. Insufficient sampling also underestimates fluctuations. Alternatively, when mixing is incomplete the suspension is heterogeneous with the average particle count in one region varying significantly from the average particle count in another. Strings of particles are observed to extend along streamlines in an incomplete mixing by stretching and folding. These heterogeneities produce increased number fluctuations at least to the same spatial scale. Similarly, uneven sample illumination produces artificial fluctuations in particle count from one side of the cell to the other. Again, the measured number fluctuations increase. Measuring Poisson statistics for the "initially randomized" suspension proved challenging but led to refinement of sample illumination, particle identification, and sample mixing. Indeed, measurement of Poisson statistics for well-mixed samples indicates proper experimental technique.

For samples having uniform density and a pair correlation with translational invariance, the (nonequilibrium) number occupancy fluctuations in a finite test volume are related to the pair correlation function or structure factor as [24]

$$
\frac{\left\langle\Delta N^{2}\right\rangle}{\langle N\rangle}=1+\frac{\phi}{v_{p}} \int H(\bar{r})[g(\bar{r})-1] d^{3} r=1+\frac{1}{(2 \pi)^{3}} \int[\theta(\bar{q})]^{2}[S(\bar{q})-1] d^{3} q
$$

where $\theta(\bar{r})$ equals unity inside the test volume and zero otherwise, $\theta(-\bar{q})$ is the Fourier transform and $H(\bar{r})=\int \theta\left(\bar{r}^{\prime}\right) \theta\left(\bar{r}-\bar{r}^{\prime}\right) d^{3} r^{\prime}$ is the convolution. Alternatively, the limits of integration may be restricted to the test volume dimension with the origin of $g(r)$ equally likely at any position. Interestingly, the pair correlation function and static structure factor are known for an equilibrium collection of hard spheres 
in the Percus-Yevick approximation. For a spherical test volume containing an average number of particles $\langle N\rangle$, we find that the approximate relationship

$$
\left\langle\Delta N^{2}\right\rangle /\langle N\rangle \approx S(0)+2[1-S(0)]\langle N\rangle^{-1 / 3}
$$

describes the exact results shown for the smaller volume fractions in Fig. 5. In the small test volume limit, the normalized mean squared number fluctuations must tend to unity, $\left\langle N^{2}\right\rangle /\langle N\rangle \rightarrow 1$. In the large test volume limit, the thermodynamic result $S(0)$ obtains. The $\langle N\rangle^{-1 / 3}$ dependence here originates, not in some exotic particle statistics, but in aliasing due to the test volume boundaries. For a spherical test volume, the function $H(r)=1-\frac{3}{4} \frac{r}{R}+\frac{1}{16}\left(\frac{r}{R}\right)^{3}$ for $\mathrm{r}<2 \mathrm{R}$ and is zero otherwise. The $\langle N\rangle^{-1 / 3}$ decay may be traced to the second term in the $H(r)$ function. This decay should not be confused with (and may indeed obscure) true $\langle N\rangle^{-1 / 3}$ number fluctuations. For the small volume fractions used in these experiments, the hard sphere $S(0)$ is within a few per cent of unity, and this model predicts very little deviation from unity for the normalized mean-square number fluctuations. Larger volume fractions produce more significant $\langle N\rangle$ dependences that should be time-independent.

Alternatively, we assume a simple model Gaussian pair correlation function:

$$
g(\bar{r})=1-[1-S(0)] \frac{\operatorname{Exp}\left[-\left(x^{2} \omega^{2 / 3}+y^{2} \omega^{2 / 3}+z^{2} \omega^{-4 / 3}\right) / \xi^{2}\right] v_{p}}{\pi^{3 / 2} \phi \xi^{3}}
$$

that gives $S(0)$ (nonthermodynamic) number fluctuations in the large test volume limit, represents a vertical to horizontal anisotropy proportional to $\omega$, can be evaluated easily in spherical (when isotropic) or rectangular coordinates, and like hard spheres has finite correlation range $\xi$. For a rectangular test volume with dimensions $[X, Y, Z]$, the number fluctuations become

$$
\left\langle\Delta N^{2}\right\rangle /\langle N\rangle=1-[1-S(0)] f\left(\omega^{1 / 3} X / \xi\right) f\left(\omega^{1 / 3} Y / \xi\right) f\left(\omega^{-2 / 3} Z / \xi\right)
$$

The function $f(t)=\operatorname{Erf}(t)-\left(1-e^{-t^{2}}\right) /(\sqrt{\pi} t)$ and ranges between zero as $t \rightarrow 0$ and unity as $t \rightarrow \infty$. If any one of the test volume dimensions tends to zero (or a small finite value), the number fluctuations tend to unity and are not suppressed (or not fully suppressed) by increasing the other test volume dimensions without limit. Figure 4 shows data compared to this functional form using experimental test volume dimensions and $\omega=2$. Fits at $2 \mathrm{~min}, 4 \mathrm{~h}$, and $8 \mathrm{~h}$ with $S(0)=0$ give $\xi / a=200,33$, and 13 , respectively. Allowing $S(0)<0$ gives a fit to the data at $6 \mathrm{~h}$ with $S(0)=-7, \xi / a=68$ and at $8 \mathrm{~h}$ with $S(0)=-0.53, \xi / a=20$. Since $g(0)=0.92$ for $\xi / a=13$, increasing to 0.98 for $\xi / a=20$, and becoming

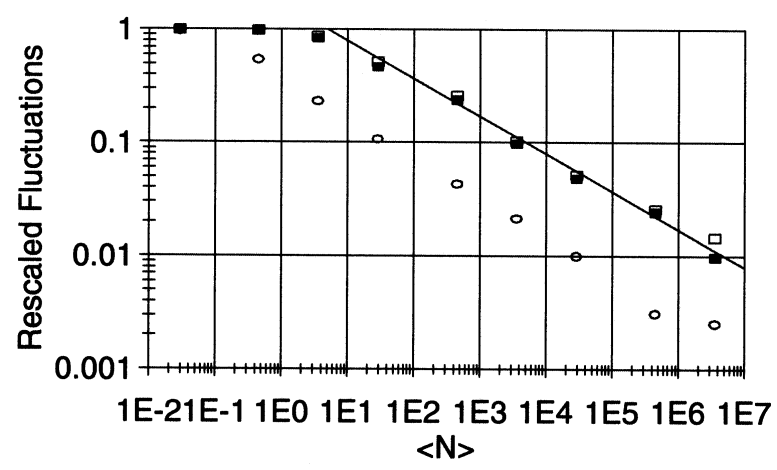

Fig. 5 The rescaled number fluctuations $\left[\left\langle\Delta N^{2}\right\rangle /\langle N\rangle-S(0)\right] /[1-S(0)]$ for hard spheres determined from the Percus-Yevick solution and plotted against the average occupancy $\langle N\rangle$. The particle volume fractions correspond to 0.01 (open squares), 0.05 (closed squares), and 0.45 (open circles). The solid line represents eq. 8 . 
essentially unity for larger values of $\xi / a$, the pair correlation may not prove measurably different from unity. Note that the theory with $S(0)=0$ approaches a limiting value $\left\langle\Delta N^{2}\right\rangle /\langle N\rangle \rightarrow 0.39$ (not zero) when increasing the average particle number by increasing viewing radius $X=Z \approx \sqrt{\pi R^{2}}$, because the light sheet thickness becomes the limiting dimension for the test volume.

The nonequilibrium-settling system is not a thermodynamic system, however, and the meaning of such results remains unclear. The correlation length of our phenomenological pair correlation function is finite and smaller than the test volume dimension for the later times. Evidently, several correlation regions are being measured, yet statistical independence does not hold. Alternatively, it is possible that the whole suspension becomes correlated. A single correlation region forms due to the long-ranged hydrodynamic interactions, and statistical independence does not apply.

Finally, Fig. 6 shows $\left\langle\Delta N^{2}\right\rangle /\langle N\rangle$ for a rectangular rather than circular test area with a horizontal to vertical length ratio, $f=X / Z$. Holding the product $X Z$ and the light sheet thickness $Y$ constant fixes the sample volume for a test of spatial anisotropy. If the number fluctuations are isotropic, the data will be symmetric when plotted against the variable $(1-f) /(1+f)$. The dashed curve gives the prediction for the isotropic $(\omega=1)$ Gaussian pair correlation function, and the solid curve is for $\omega=2$. The data is not symmetric, and the $\omega=2$ curve gives a slightly better fit than the isotropic curve. The anisotropy develops over a long period of time and evidences a correlation length approximately two times larger in the vertical direction. This should be compared with the dynamical anisotropy observed for velocity fluctuations $[8,14]$ and anticipated in simulations[25] and theoretically $[17,26]$.

Initially, well-mixed samples evidence Poisson number statistics. This is accompanied by large velocity fluctuations. In time, the number and velocity fluctuations become suppressed $[21,27,28]$. The correlation between number statistics and velocity fluctuations suggests that more-dense regions of particles tend to move downward and less-dense regions upward to produce observed velocity fluctuations. It is possible that this leads to a slight stratification of the suspension having a concentration gradient from top to bottom. Such a gradient is postulated as a mechanism to quench velocity fluctuations [19]. Alternatively, the experimental samples have finite vertical extent and density fluctuations may simply leave the sample, the larger, more mobile ones first. When this happens the sample becomes progres-

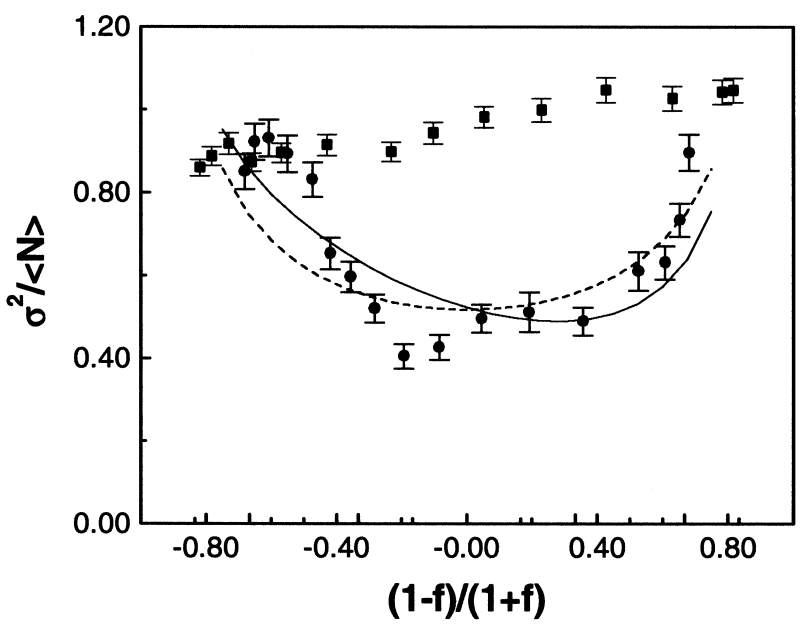

Fig. 6 Reduced number fluctuations, $\left\langle\Delta N^{2}\right\rangle /\langle N\rangle=\sigma^{2} /\langle N\rangle$ as a function of sample volume anisotropy, $(1-f) /(1+f)=(Z-X) /(Z+X)$ with $\langle N\rangle=30$ at 2 min after mixing (closed squares) and $8 \mathrm{~h}$ after mixing (closed circles). The dashed line represents $\xi / a=13, \omega=1$ (isotropic), and the solid line is for $\xi / a=13, \omega=2$. The error bars represent $68 \%$ confidence limits of the average value. 
sively more uniform and number fluctuations are reduced. Since the larger-scale fluctuations of the random system are winnowed out, the suspension becomes subtly ordered. Clearing the fluctuations from the sample or forming a density gradient should depend on cell height. Indeed, preliminary measurements of both velocity fluctuations [27] and number fluctuations [29] demonstrate that longer cells evidence slower evolution of velocity and number fluctuations. For example, the same degree of number fluctuation suppression for test volumes with $\langle N\rangle=100$ in a 4-cm-tall cell vs. a $23-\mathrm{cm}$-tall cell is $8 \mathrm{~h}$ and $71 \mathrm{~h}$, respectively, a rough scaling with cell height. If the experimental suppression of fluctuations is due to one of these mechanisms, then the paradox of Calflish and Luke remains for very tall to infinitely tall samples, because fluctuations never clear the sample or a concentration gradient is never fully achieved.

\section{ACKNOWLEDGMENT}

We thank NASA for supporting this work through Grant No. NAG3-1852.

\section{REFERENCES}

1. L. D. Landau and E. M. Lifschitz. Statistical Physics p. 6ff. Addison-Wesley, Reading, MA (1970).

2. R. M. Lynden-Bell, J. P. Noworyta, J. Rasaiah. Pure Appl. Chem. 73, 1721-1731 (2001).

3. C. A. Angell. Ser. Dir. Condens. Matter Phys. 17, 151 (2000).

4. P. N. Pusey and W. Van Megen. Physica A (Amsterdam) 157, 705-741 (1989).

5. R. E. Caflisch and J. H. C. Luke. Phys. Fluids 28, 759-760 (1985).

6. G. K. Batchelor. J. Fluid Mech. 52, 245-268 (1972).

7. M. P. Brenner. Phys. Fluids 11, 754-772 (1999).

8. P. N. Segre, E. Herbolzheimer, P. M. Chaikin. Phys. Rev. Lett. 79, 2574-2577 (1997).

9. E. J. Hinch. In Disorder and Mixing, E. Guyon (Ed.), pp. 153-161, Kluwer, Dordrecht (1988).

10. A. J. C. Ladd. Phys. Rev. Lett. 76, 1392-1395 (1996).

11. A. J. C. Ladd. Phys. Fluids 9, 491-499 (1997).

12. J. M. Ham and G. M. Homsy. Int. J. Multiphase Flow 14, 533-546 (1988).

13. H. Nicolai and E. Guazzelli. Phys. Fluids 7, 3-5 (1995).

14. H. Nicolai, B. Herzhaft, E. J. Hinch, L. Oger, E. Guazzeli. Phys. Fluids 7, 12-23 (1995).

15. D. L. Koch and E. S. G. Shaqfeh. J. Fluid Mech. 224, 275-303 (1991).

16. P. Tong and B. J. Ackerson. Phys. Rev. E 58, 6931-6934 (1998).

17. A. Levine, S. Ramaswamy, E. Frey, R. Bruinsma. Phys. Rev. Lett. 81, 5944-5947 (1998).

18. E. Asmolov. J. Fluid Mech.(2002) Submitted for publication.

19. J. H. C. Luke. Phys. Fluids 12, 1619-1621 (2000).

20. T. N. Smith. J. Fluid Mech. 32, 203-207 (1968).

21. X. Lei, B. J. Ackerson, P. Tong. Phys. Rev. Lett. 86, 3300-3303 (2001).

22. W. B. Russel, D. A. Saville, W. R. Schowalter. Colloidal Dispersions, Chap. 12, Cambridge University Press, Cambridge (1989).

23. M. Wertheim. Phys. Rev. Lett. 10, 321-323 (1963); E. Thiele. J. Chem. Phys. 38, 1959-1966 (1963).

24. B. J. Berne. In Photon Correlation Spectroscopy and Velocimetry, H. Z. Cummins and E. R. Pike (Eds.), pp. 344-385, Plenum Press, New York (1976).

25. A. J. C. Ladd. Phys. Fluids 5, 299-310 (1993).

26. D. L. Koch. Phys. Fluids 5, 1141-1155 (1993).

27. S. Tee, E. R. Weeks, D. A. Weitz. Bull. A.P.S. 45, 70 (2000).

28. E. Guazzelli. Phys. Fluids 13, 1537-1540 (2001).

29. X. Lei, B. J. Ackerson, P. Tong. Bull. A.P.S. 45, 70 (2000). 